\title{
Crowd-Driven Processes: State of the Art and Research Challenges
}

\author{
Maja Vukovic ${ }^{1}$ and Claudio Bartolini ${ }^{2}$ \\ 1 IBM Research, 19 Skyline Dr, Hawthorne, NY 10532, USA \\ maja@us.ibm.com \\ 2 HP Labs, 1501 Page Mill Road, Palo Alto, CA 94304, USA \\ claudio.bartolini@hp.com
}

\begin{abstract}
Over the past few years the crowdsourcing paradigm has evolved from its humble beginnings as isolated purpose-built initiatives, such as Wikipedia and Elance and Mechanical Turk to a growth industry employing over 2 million knowledge workers, contributing over half a billion dollars to the digital economy. Web 2.0 provides the technological foundations upon which the crowdsourcing paradigm evolves and operates, enabling networked experts to work collaboratively to complete a specific task.

Crowdsourcing has a potential to significantly transform the business processes, by incorporating the knowledge and skills of globally distributed experts to drive business objectives, at shorter cycles and lower cost. Many interesting and successful examples exist, such as GoldCorp, TopCoder, Threadless, etc. However, to fully adopt this mechanism enterprises, and benefit from appealing value propositions, in terms of reducing the time-to-value, a set of challenges remain, in order for enterprises to retain the brand, achieve high quality contributions, and deploy crowdsourcing at the minimum cost.

Enterprise crowdsourcing poses interesting challenges for both academic and industrial research along the social, legal, and technological dimensions. In this tutorial we present a landscape of existing crowdsourcing applications, targeted to the enterprise domain. We describe the challenges that researchers and practitioners face when thinking about various aspects of enterprise crowdsourcing. First, to establish technological foundations, what are the interaction models and protocols between the Enterprise and the crowd (including different types of crowd, such as internal, external and hybrid models). Secondly, how is crowdsourcing going to face the challenges in quality assurance, enabling Enterprises to optimally leverage the scalable workforce. Thirdly, what are the novel (Web) applications enabled by Enterprise crowdsourcing, and how can existing business processes be transformed for crowd consumption.
\end{abstract}

